\title{
Perfil comunicativo de indivíduos com a síndrome de Williams-Beuren
}

\section{Communicative profile of individuals with Williams-Beuren syndrome}

\author{
Natalia Freitas Rossi ${ }^{1}$, Danilo Moretti-Ferreira ${ }^{2}$, Célia Maria Giacheti ${ }^{3}$
}

\begin{abstract}
RESUMO
Objetivo: Descrever o perfil comunicativo de indivíduos com a síndrome de Williams-Beuren. Métodos: A casuística foi composta por 12 indivíduos com a síndrome com idade cronológica entre 6;6 a 23;6 (Grupo 1) que foram comparados a outros 12 sem a síndrome e com idade mental semelhante e sem dificuldades de linguagem/aprendizagem (Grupo 2). Os indivíduos foram avaliados em situação de conversação para classificação dos comportamentos verbais e não-verbais, segundo critérios pragmáticos, levantamento do número de turnos por minuto, enunciados por turno, Extensão Média de Enunciados, levantamento quanto à frequiência e tipologia de disfluências da fala e classificação quanto ao tipo de pausas plenas do discurso. Resultados: $\mathrm{O}$ perfil comunicativo do Grupo 1 mostrou facilidade para interagirem em situação de comunicação com a presença de limitações lingüísticas estruturais e funcionais variáveis, quando comparados aos indivíduos do Grupo 2. Os indivíduos do Grupo 1 freqüentemente utilizaram estratégias comunicativas, na tentativa de preencherem o espaço comunicativo, como o uso de clichês, efeitos sonoros, recursos entonacionais e as pausas plenas que mostraram ser favoráveis do ponto de vista sócio-comunicativo, enquanto que os comportamentos verbais ecolálicos e perseverativos prejudicam o desempenho comunicativo desses indivíduos. Conclusão: $\mathrm{O}$ desempenho comunicativo mais prejudicado do Grupo 1 permitiu especular que comprometimentos lingüísticos nesta condição podem estar presentes, independente da diferença entre idade cronológica e mental. Estudos mais abrangentes poderão responder ao questionamento da dissociação de habilidades cognitivas e lingüísticas nesta síndrome e também no que diz respeito à complexa esta correlação em meio aos distúrbios da comunicação humana.
\end{abstract}

DESCRITORES: Cognição; Comunicação; Linguagem; Síndrome de Williams

\section{INTRODUÇÃO}

A síndrome de Williams-Beuren (SWB) tem sido objeto de estudo no que concerne à compreensão dos mecanismos

(1) Pós-graduanda em Ciências Biológicas (Genética) do Instituto de Biociências da Universidade Estadual Paulista - UNESP - Botucatu (SP), Brasil.

(2) Professor Doutor do Instituto de Biociências da Universidade Estadual Paulista - UNESP - Botucatu (SP), Brasil; Chefe do Serviço de Aconselhamento Genético (SAG) do Instituto de Biociências da Universidade Estadual Paulista - UNESP - Botucatu (SP), Brasil.

(3) Professora Livre Docente do Curso de Fonoaudiologia da Faculdade de Filosofia e Ciências da Universidade Estadual Paulista - UNESP - Marília (SP), Brasil; Orientadora do Programa de Pós-Graduação em Ciências Biológicas (Genética) do Instituto de Biociências da Universidade Estadual Paulista - UNESP - Botucatu (SP), Brasil.

Trabalho realizado no Serviço de Aconselhamento Genético (SAG) do Instituto de Biociências da Universidade Estadual Paulista - UNESP Botucatu (SP), Brasil, com auxílio financeiro do Conselho Nacional de Desenvolvimento Científico e Tecnológico - CNPq. Este trabalho faz parte da dissertação de mestrado apresentada ao Programa de Pós-Graduação em Ciências Biológicas (Genética) do Instituto de Biociências da Universidade Estadual Paulista - UNESP - Botucatu (SP), Brasil.

Endereço para correspondência: Natália Freitas Rossi. R. Jundiaí, 1215, Jardim Buscardi, Matão - SP, CEP 15990-510. E-mail: nataliafreitasrossi@yahoo.com.br

Recebido em: 18/9/2006; Aceito em: 15/2/2007 genéticos e neurobiológicos que influenciam os comportamentos sociais, as habilidades neuropsicológicas e lingüísticas. Neste sentido, muitos pesquisadores têm investido nos estudos de correlação genótipo-fenótipo para melhor entender os aspectos comportamentais, cognitivos, lingüísticos e suas bases moleculares ${ }^{(1)}$.

Esta síndrome ocorre em um a cada 7.500 recém nasci$\operatorname{dos}^{(2)}$ e sua etiologia está associada a uma microdeleção que ocorre na região cromossômica 7q11.23. Esta deleção ocasiona a perda de aproximadamente 20-25 genes e, por isso, determina um fenótipo variável. O fenótipo da SWB inclui aspecto facial típico caracterizado pela presença de bochechas proeminentes, narinas antevertidas, filtro nasal longo, proeminência periorbitária e boca grande com lábios volumosos. Apresenta, também, alterações cardíacas, sendo a mais comum a estenose aórtica supravalvular, além de um conjunto de manifestações cognitivas e de linguagem que resultam em comportamentos comunicativos e sociais bastante peculiares a esta síndrome ${ }^{(3)}$.

O perfil neuro-cognitivo desses indivíduos é descrito como "picos e vales" pela excelência no funcionamento lingüístico e social em detrimento das dificuldades cognitivas, principalmente de funções viso-construtivas ${ }^{(4)}$. A deficiência mental é uma manifestação comum à síndrome, sendo o grau de comprometimento variável de leve a moderado ${ }^{(5)}$. 
A característica sociável e falante que os indivíduos com a SWB apresentam conferiu-lhes a denominação da síndrome Cocktail Party Speech $(C P S)^{(6)}$. As características CPS incluem comportamento falante, fala fluente com precisão articulatória, uso de frases estereotipadas e de clichês, hábito de inserir experiências pessoais e irrelevantes ao contexto, além de comportamentos perseverativos. Os autores destacaram que este perfil comunicativo, obtido a partir de uma amostra de conversação, não é comum a todos os indivíduos com a SWB, havendo, portanto variabilidade entre os casos.

A característica de fala inteligível descrita nesta síndrome é justificada pela participação efetiva do circuito fonológico, garantindo a esses sujeitos a retenção e a manipulação de informações fonológicas acopladas ao componente fonoarticulatório. A integridade do sistema fonológico, como parte das habilidades de linguagem nesta síndrome, se sustenta pelos estudos que mostraram desempenho semelhante entre os indivíduos com a SWB com o de indivíduos de mesma idade mental e com desenvolvimento de linguagem típico $^{(7-8)}$. Porém, outros estudos que também compararam o desempenho dos indivíduos com a síndrome a outros de mesma idade mental e com ausência de dificuldades de linguagem, mostraram que tanto o processamento semântico quanto o fonológico podem estar prejudicados na $\mathrm{SWB}^{(9-10)}$.

Já, no que diz respeito à característica de fala fluente, atribuída aos indivíduos com a SWB, autores discordaram desta qualidade quando compararam o desempenho desses indivíduos a outros de mesma idade mental, com desempenho lingüístico dentro dos padrões de normalidade e que identificaram a presença de prejuízos na fluência verbal decorrente de dificuldades na seleção lexical. Esses achados mostraram que a fluência verbal dos indivíduos com a SWB frequientemente está prejudicada em decorrência dos prejuízos léxico-semânticos, principalmente em situações que requerem a nomeação verbal e em situações de produção espontânea da fala ${ }^{(4,7-8)}$.

Embora discordantes vários estudos têm sido realizados na tentativa de estudar a competência sintática desses indivíduos. Neste contexto, algumas pesquisas que compararam o desempenho desses ao de outros de mesma idade mental e com ausência de dificuldades de linguagem, revelaram que os indivíduos com a SWB apresentaram desempenho adequado nas tarefas que avaliaram a habilidade sintática ${ }^{(4)}$, enquanto que outras mostraram algum nível de comprometimento sintático, no que se refere às dificuldades para domínios mais complexos ${ }^{(5,10-12)}$.

A suposta dissociação de aspectos cognitivos e lingüísticos é um ponto ainda bastante controvertido na literatura. Autores afirmaram que a idéia de dissociação é pouco provável se for levada em consideração a complexidade de tarefas lingüísticas, como é o caso dos aspectos lingüístico-narrativos, sendo possível constatar a presença de prejuízos lingüísticos graves para esses indivíduos quando comparados a outros de mesma idade mental e com ausência de dificuldades de linguagem $^{(13)}$.

Vários estudos foram realizados com a SWB focando características cognitivas e lingüísticas. No campo da linguagem, grande parte das pesquisas foram efetuadas, no sen- tido de avaliar habilidades sintática, semântica e fonológica. Porém, poucos foram os estudos ${ }^{(5,14-18)}$ que se detiveram especificamente na avaliação da linguagem sob uma perspectiva pragmática, principalmente com enfoque nas habilidades conversacionais.

Dificuldades pragmáticas e sócio-comunicativas foram apontadas para os indivíduos com a SWB, em estudo, no qual os pesquisadores realizaram várias comparações estatísticas entre os indivíduos com a SWB, indivíduos com a síndrome de Down, indivíduos com Distúrbio Específico de Linguagem e indivíduos com desenvolvimento típico de linguagem (DT). O estudo mostrou que a característica falante dos indivíduos com a SWB contribui para as transgressões, uso de regras sócio-comunicativas, uma vez que tais indivíduos não se inibem perante interlocutores desconhecidos. Os autores notaram que a dificuldade conversacional apresentada pelos indivíduos com a SWB foi comparada aos prejuízos lingüísticos típicos dos distúrbios semântico-pragmáticos da linguagem, observados nos indivíduos com Distúrbio Específico de Linguagem ${ }^{(14)}$.

Se, por um lado, estudos apontam para dificuldades lingüísticas nesta síndrome, por outro, o uso de recursos comunicativos como a prosódia parece refletir na "aparente" competência comunicativa dos indivíduos com a SWB ${ }^{(15-17)}$. Esses recursos conferem-lhes um estilo comunicativo característico dos contadores de histórias, pelo uso excessivo de marcadores de entonação, mas que na realidade, apresentam dificuldades significativas quanto à coerência e complexidade da estrutura narrativa. Segundo os autores, esses achados puderam ser observados uma vez que a estratégia de avaliação priorizou a narrativa oral ${ }^{(15)}$.

A investigação da habilidade prosódica em indivíduos com a SWB foi focada recentemente em um estudo que mostrou a presença de alterações nesta habilidade que interferem na competência comunicativa desses indivíduos ${ }^{(18)}$. Parte dos autores que realizaram o estudo anterior publicou outro trabalho que reforça a presença de competências comunicativas variáveis entre os indivíduos com a SWB e que, portanto a tentativa de homogeneizar o perfil comunicativo desses indivíduos como favorável em detrimento às limitações cognitivas, seria decorrente dos vários pontos de vista teóricos e metodológicos que devem ser debatidos ${ }^{(19)}$. Em ambos os estudos, os indivíduos com a SWB foram avaliados em situação de conversação, estratégia esta, justificada pelos autores como um método que permite obter dados mais próximos a real competência lingüística dos indivíduos.

A presença de heterogeneidade não se restringe às manifestações lingüísticas e, também, pode ser encontrada nas avaliações cognitivas. Estudo recente mostrou que os indivíduos com a SWB podem apresentar funções cognitivas com maior ou menor grau de comprometimento e que, portanto, não há uma linearidade de manifestações a este respeito ${ }^{(20)}$.

$\mathrm{O}$ relato de caso de uma menina com 10 meses de idade, diagnosticada com a SWB, mostrou a presença de comprometimento nos aspectos sócio-comunicativos da linguagem $^{(19)}$, enfatizando que a investigação da linguagem sob o foco da pragmática nesta síndrome é fundamental. Sob esta mesma perspectiva ${ }^{(17)}$, foi demonstrado que a "aparente" com- 
petência lingüística apresentada por esses indivíduos está relacionada ao uso de estratégias conversacionais, as quais não podem ser observadas quando os mesmos são avaliados pelos testes padronizados.

Estudo recente destacou a necessidade dos profissionais, que atuam na área de avaliação da linguagem, de complementarem seus achados com o uso de procedimentos que, embora não estandardizados, permitem uma visão mais detalhada no âmbito pragmático, social e funcional das habilidades comunicativas, principalmente em indivíduos com alterações intelectuais ${ }^{(21)}$.

Considerando a divergência entre os estudos que abordaram as habilidades de linguagem e a escassez de estudos sobre habilidades pragmáticas na SWB, este trabalho tem por objetivo investigar o perfil comunicativo de indivíduos com esta síndrome. A hipótese levantada para este estudo é da existência de diferenças importantes no desempenho comunicativo dos indivíduos com a SWB, quando comparados a outros de mesma idade mental com ausência de dificuldades de linguagem/aprendizagem. O estudo comparativo das habilidades de linguagem de indivíduos com a SWB, equiparados a outros de mesma idade mental, com desenvolvimento de linguagem típico, tem sido uma metodologia freqüentemente utilizada pelos estudos internacionais ${ }^{(4,6-13)}$ na tentativa de aproximar ao máximo o nível intelectual dos indivíduos, para que os aspectos lingüísticos pudessem ser melhor investigados e discutidos, até mesmo para debater a hipótese de dissociação entre as habilidades cognitivas e de linguagem nesta síndrome. Desta forma, a comprovação da hipótese levantada contribuiria para a visão da heterogeneidade lingüística entre esses indivíduos, com base no ponto de vista teórico da avaliação. Pretende-se mostrar, também, com este estudo que o uso de uma estratégia avaliativa que privilegia uma análise funcional da linguagem poderá apontar para diferentes achados que contribuirão no processo de intervenção e melhor adequação sócio-comunicativa desses indivíduos.

Assim, neste estudo, optou-se pelo uso da estratégia de conversação que revela incrível sensibilidade à detecção de dificuldades pragmáticas ${ }^{(22)}$ e que, também, possibilita investigar o perfil comunicativo nesta síndrome, conforme mostraram estudos internacionais que abordaram aspectos da linguagem dos indivíduos com a SWB a partir da conversação ${ }^{(5,14,17,19)}$.

\section{MÉTODOS}

Este estudo foi aprovado pelo Comitê de Ética em Pesquisa (CEP) da Faculdade de Medicina de Botucatu, registro $\mathrm{n}^{\circ} 1238-2003$ e processo $\mathrm{n}^{\circ} 25000.057294 / 2003-23$ e pelo Conselho Nacional de Ética em Pesquisa (CONEP), registro $n^{\circ} 7834$ e parecer $n^{\circ} 1245-03$. Os responsáveis pelos indivíduos autorizaram sua participação, mediante assinatura do Termo de Consentimento Livre e Esclarecido, elaborado para fins específicos desta pesquisa, segundo resolução do Conselho Nacional de Saúde, CNS/196/96 ${ }^{(23)}$ sobre Diretrizes e Normas Regulamentadoras de Pesquisas Envolvendo Seres Humanos e recomendações dos relatores CEP e CONEP.

\section{Casuística}

A casuística deste estudo foi constituída por 24 indivíduos, subdivididos em dois grupos. O Grupo 1 foi formado por 12 indivíduos com diagnóstico clínico e laboratorial da síndrome de Williams-Beuren (SWB). O Grupo 2 foi composto por 12 indivíduos de mesmo gênero e idade mental semelhante à do Grupo 1. Estes indivíduos não apresentavam sinais clínicos da síndrome de Williams-Beuren, não apresentavam queixas neurológicas e tinham histórico de desenvolvimento típico de linguagem/aprendizagem, segundo informações dos pais e professores.

A idade mental e o gênero foram os critérios utilizados por este estudo para fins de comparação estatística entre os grupos. A idade mental foi obtida a partir da avaliação de desempenho intelectual que utilizou a escala Wechsler para crianças $^{(24)}$, para pré-escolares ${ }^{(25)}$ e para adultos ${ }^{(26)}$.

Os indivíduos do Grupo 1 foram selecionados a partir dos bancos de dados do Serviço de Aconselhamento Genético - Departamento de Genética da UNESP de Botucatu/SP. Constituíram critérios de inclusão para este grupo a confirmação diagnóstica da SWB a partir da avaliação genética médica e resultado citogenético-molecular positivo para a deleção do gene da Elastina (ELN) na região 7q11.23, investigado pela técnica de Hibridização in situ por Fluorescência (FISH). Os procedimentos de avaliação da linguagem foram inicialmente aplicados nos 23 indivíduos que apresentavam características clínicas condizentes com o fenótipo da SWB. Destes, foram selecionados apenas os 12 indivíduos que obtiveram confirmação diagnóstica clínica e citogenéticamolecular da SWB. Dos 12 indivíduos, seis eram do gênero masculino e seis do gênero feminino com idade cronológica entre 6,6 e 23,6 anos (média de 12,3 anos) e idade mental entre 4,7 e 14,2 anos (média de 8,5 anos). Cinco dos 12 indivíduos freqüentavam ensino regular, sendo que dentre os cinco, quatro estavam em processo inicial de alfabetização e apenas um estava alfabetizado e cursando a $5^{\text {a }}$ série do ensino regular. Um indivíduo concluiu o ensino médio e um deles não frequientou o ensino formal. Os demais indivíduos (5/12) freqüentam ensino especial na Associação de Pais e Amigos dos Excepcionais (APAE) do Município de origem.

Para constituir o Grupo 2 foram selecionados indivíduos voluntários, estudantes da rede municipal do ensino regular, os quais foram submetidos inicialmente à avaliação psicométrica para determinar a idade mental. Esses indivíduos foram pré-selecionados pelos professores, segundo critérios de ausência de queixas de dificuldades de linguagem/ aprendizagem e de história pregressa de tratamentos fonoaudiológicos ou pedagógicos. Histórico de desenvolvimento de linguagem/aprendizagem e queixas neurológicas foi coletado com pais/responsáveis. Submeteram-se à avaliação psicométrica 22 indivíduos com faixa etária entre 04 a 16 anos de idade e escolaridade entre o Pré-I ao $1^{\circ}$ colegial. Dos 22 indivíduos submetidos aos testes psicométricos foram selecionados 12 que compuseram o Grupo 2 e que também foram submetidos aos procedimentos de avaliação da linguagem, segundo o gênero e idade mental para fins de comparação estatística entre os grupos. 


\section{Procedimentos}

Os indivíduos de ambos os grupos foram avaliados em situação de conversação, que ocorreu individualmente com o avaliador e registrada em filmadora da marca SONY CCDTRV11E $8 \mathrm{~mm}$.

Os indivíduos foram filmados por aproximadamente 40 minutos, sendo estabelecido um tempo médio de filmagem comum aos indivíduos de ambos os grupos, para garantir a unidade quanto ao tempo da amostra de conversação analisada.

Para este estudo foram transcritos e analisados 17 minutos de conversação de cada indivíduo e foram consideradas as seguintes medidas obtidas a partir da transcrição da amostra:

1) classificação dos comportamentos comunicativos verbais e não-verbais como adequados ou inadequados, segundo Proposta do Protocolo de Avaliação da Pragmática ${ }^{(27)}$ que considera aspectos relacionados ao: turno e tópico comunicativo, seleção lexical e aspectos paralingüísticos. O julgamento do comportamento comunicativo e a classificação como adequado ou inadequado foram realizados pelo próprio avaliador e por outro profissional experiente na área de avaliação da linguagem. Foram considerados recursos comunicativos inadequados quando o recurso comunicativo verbal ou não-verbal utilizado pelo indivíduo ocasionou em quebra no processo comunicativo entre os interlocutores e o conceito contrário foi aplicado para classificar o ato comunicativo como adequado. A partir da classificação dos comportamentos comunicativos foi realizada a comparação entre a freqüência de iniciativas e respostas verbais inadequadas. Também foi analisado o uso de recursos que, segundo a literatu$\mathrm{ra}^{(15-17)}$, favorecem o processo comunicativo dos indivíduos com a SWB, do ponto de vista funcional, como o uso de recursos com efeito sonoro e o uso de moduladores entonacionais (altura melódica, tempo e tensão fonêmica).

2) levantamento do número de turnos por minuto, enunciados por turnos e a extensão média de enunciados (EME) que corresponde ao número de palavras por enunciado. Essas medidas foram utilizadas, pois permitem visualizar aspectos importantes do ponto de vista funcional e estrutural da linguagem.

3) classificação quanto ao tipo de pausa plena (partículas de codificação e de repetição) e frequiência de ocorrência. A frequiência de ocorrência de pausas foi obtida a partir do Teste de Linguagem - Área da Fluência ${ }^{(28)}$. Foi realizado o levantamento referente à tipologia das disfluências, que correspondeu ao número de ocorrências para cada tipo de disfluências (disfluências comuns - hesitações; interjeições; revisões; palavras não terminadas, repetições de segmentos e repetições de frases e disfluências gagas - duas ou mais repetições de sons e/ou sílabas, e/ou palavras; prolongamentos; bloqueios, pausas e intrusão) e referente à freqüência de rupturas da fala (analisada pela porcentagem de descontinuidade de fala a partir do número total de rupturas comuns e gagas) e a porcentagem de disfluências gagas (taxa de rupturas gagas).

Para a análise estatística comparativa entre os dois grupos foram empregados testes de distribuição livre (ou não- paramétricos). No caso da variável em estudo ser numérica, utilizou-se o teste de Mann-Whitney. Para avaliar a existência de associação entre dois ou mais grupos quanto às variáveis categóricas foi utilizado o teste exato de Fisher. Os níveis de significância adotados foram de $\mathrm{p}<0,05$ (*) e $\mathrm{p}<0,01$ (**).

\section{RESULTADOS}

Os resultados deste estudo apontaram para a presença de características comunicativas recorrentes entre os indivíduos com a SWB, mas distintas quanto ao grau de comprometimento.

A análise estatística comparativa entre os grupos de idade mental semelhantes mostrou diferenças relevantes quanto aos aspectos da linguagem, no que diz respeito aos comportamentos comunicativos lingüísticos e paralingüísticos.

A Tabela 1 mostra a presença dos comportamentos comunicativos analisados e julgados como adequados ou inadequados dos grupos, referentes ao uso de habilidades lingüísticas e paralingüísticas.

A intenção de realizar um ato comunicativo foi observada em ambos os grupos, não caracterizando diferenças estatisticamente significantes entre eles. Porém, tal diferença foi notável com relação ao uso dos recursos comunicativos verbais e não-verbais para concretizar essas intenções na comparação de desempenho entre os grupos estudados. Enquanto os indivíduos do Grupo 1 apresentaram freqüência elevada para a ocorrência de iniciativas e respostas inadequadas, tais aspectos não foram observados para os indivíduos do Grupo 2, justificando o baixo valor-p encontrado $(\mathrm{p}<0,001)$.

Quando realizada a comparação estatística intra-grupo entre os valores percentuais de iniciativas e respostas inadequadas do Grupo 1, foi possível verificar uma maior ocorrência de iniciativas inadequadas se comparada às respostas inadequadas apresentadas pelos indivíduos durante a conversação $(\mathrm{p}=0,013)$.

A ininteligibilidade de fala foi um achado relevante no que diz respeito tanto às limitações para iniciar quanto responder durante o processo conversacional, como mostrou a Tabela 1.

Dentre os comportamentos que contribuíram para o aumento de iniciativas inadequadas, observou-se a presença de enunciados ecolálicos e perseverativos e a retomada de tópico sem o uso de estratégias lingüísticas que sinalizam a reintrodução temática durante o intercâmbio conversacional. Outra análise estatística intra-grupo (Grupo 1) foi realizada para verificar quais desses comportamentos favoreceram a ocorrência de iniciativas inadequadas. Esta análise mostrou que tanto os comportamentos verbais ecolálicos quanto os perseverativos não apresentaram diferenças estatisticamente significantes ( $\mathrm{p}=0,478$ e $\mathrm{p}=0,217$ ), o que mostra não ser característica comum a todos os indivíduos do grupo com a SWB, ao contrário do que foi observado para a retomada frequiente de tópico $(\mathrm{p}=0,037)$.

A análise comparativa entre os grupos quanto ao uso de recursos lingüísticos e paralingüísticos, que supostamente são comuns aos indivíduos com a SWB e que favoreceram o processo comunicativo do ponto de vista funcional, mostrou ten- 
Tabela 1. Análise estatística comparativa entre G1 e G2 quanto ao uso de habilidades pragmáticas

\begin{tabular}{|c|c|c|c|c|c|c|}
\hline \multirow[b]{2}{*}{ Habilidades Pragmáticas } & \multicolumn{2}{|c|}{ G1 } & \multicolumn{2}{|c|}{ G2 } & \multirow[b]{2}{*}{$\mathrm{p}$} & \\
\hline & Inadequada & Adequada & Inadequada & Adequada & & \\
\hline \multicolumn{7}{|l|}{ Aspectos Lingüísticos } \\
\hline \multicolumn{7}{|l|}{ Turno } \\
\hline Adotar e manter turnos & 2 & 10 & 0 & 12 & 0,478 & \\
\hline Respeitar o turno do interlocutor & 7 & 5 & 0 & 12 & 0,005 & ** \\
\hline Mecanismos de reparo e revisão & 10 & 2 & 0 & 12 & $<0,001$ & ** \\
\hline Recursos de Feed-back & 7 & 5 & 0 & 12 & 0,005 & ** \\
\hline \multicolumn{7}{|l|}{ Tópico } \\
\hline Seleção & 7 & 5 & 0 & 12 & 0,005 & ** \\
\hline Introdução & 4 & 8 & 0 & 12 & 0,093 & \\
\hline Manutenção & 5 & 7 & 0 & 12 & 0,037 & * \\
\hline \multicolumn{7}{|l|}{ Aspectos lexicais } \\
\hline Coerência - linearidade semântica & 7 & 5 & 0 & 12 & 0,005 & ** \\
\hline Seqüenciar e relacionar fatos & 5 & 7 & 0 & 12 & 0,037 & * \\
\hline \multicolumn{7}{|l|}{ Aspectos Paralingüísticos } \\
\hline Inteligibilidade & 5 & 7 & 0 & 12 & 0,037 & * \\
\hline Prosódia & 2 & 10 & 0 & 12 & 0,478 & \\
\hline Fluência & 2 & 10 & 0 & 12 & 0,478 & \\
\hline
\end{tabular}

${ }^{*} \mathrm{p}<0,05{ }^{* *} \mathrm{p}<0,01$; Adaptado de Prutting, Kirchner(27)

dência à diferença estatisticamente significante no que concerne ao uso de clichês (expressões idiomáticas) (e.g. "A união faz a força"), trocadilhos e jogos verbais (e.g. "papo vai, papo vem") observados durante conversação ( $\mathrm{p}=0,093)$.

Também foi notada tendência à significância estatística quando realizada uma análise comparativa entre os grupos, no que diz respeito ao uso de moduladores entonacionais. Verificou-se que os indivíduos do Grupo 1 tendem a utilizar de forma mais enfática do que o Grupo 2 recursos como a altura melódica $(\mathrm{p}=0,069)$, aumento no tempo de fonação ("muito" pronunciado como "muuuuuuito") e aumento na tensão fonêmica ("Menina" pronunciada como "MEnina"), sendo o valor-p desses dois últimos itens de 0,089.

A Tabela 2 mostra o resultado da análise estatística comparativa entre os grupos, referente ao comportamento verbal que incluiu o levantamento da freqüência de turnos por minuto, enunciados por turno e a extensão média de enuncia- dos (EME). Nota-se, nesta tabela, a presença de diferença estatisticamente significante entre os grupos para o primeiro e o último item analisado.

A Tabela 3 mostra a caracterização das rupturas da fala que foram classificadas como pausas plenas do discurso. Quanto à utilização de pausas plenas, foi possível notar que houve diferenças significativas entre os dois grupos. Esta tabela mostra que os indivíduos do Grupo 1 apresentaram freqüência aumentada para o uso de partículas que interferem no encadeamento de enunciados, sejam elas de codificação ou de repetição.

A análise comparativa entre os grupos, referente à porcentagem de rupturas na fala, mostrou a ocorrência de diferença estatisticamente significante entre os grupos, sendo $\mathrm{p}=0,019$ para a porcentagem de descontinuidade da fala e $\mathrm{p}=0,002$ para a porcentagem de descontinuidade gaga. É importante ressaltar, nesta última análise, uma assimetria na

Tabela 2. Análise estatística comparativa entre G1 e G2 quanto aos atos comunicativos verbais

\begin{tabular}{lccccccc}
\hline & & G1 & & & G2 & & \\
Itens Analisados & Mín. & Máx. & Md & Mín. & Máx. & Md & $p$ \\
\hline Turnos / minuto & 3,2 & 12,5 & 7,6 & 1,6 & 7,5 & 4,3 & $0,005 * *$ \\
Enunciado / turno & 0,8 & 4,2 & 1,5 & 1,2 & 5,1 & 1,5 & 0,686 \\
Extensão média do enunciado (EME) & 0,6 & 3,0 & 3,0 & 3,3 & 7,5 & 4,8 & $0,002 * *$ \\
\hline
\end{tabular}

Nota: Min. = Mínimo; Max. = Máximo; Md=Mediana; * $p<0,05{ }^{* *} p<0,01$

Tabela 3. Análise estatística comparativa entre G1 e G2 quanto a ocorrência de pausas plenas identificadas durante a conversação

\begin{tabular}{|c|c|c|c|c|c|c|c|}
\hline \multirow[b]{2}{*}{ Pausas Plenas } & \multicolumn{3}{|c|}{ G1 } & \multicolumn{3}{|c|}{ G2 } & \multirow[b]{2}{*}{$p$} \\
\hline & Min. & Max. & Md. & Min. & Max. & Md. & \\
\hline Inserção de partículas de codificação & 1,0 & 30,0 & 15,5 & 2,0 & 14,0 & 8,0 & 0,018 ** \\
\hline Repetições de partículas & 0,0 & 17,0 & 4,0 & 0,0 & 3,0 & 0,0 & $0,010 * *$ \\
\hline
\end{tabular}

Nota: Min. = Mínimo; Max. = Máximo; Md=Mediana; ${ }^{*} p<0,05{ }^{* *} p<0,01$ 
distribuição de frequiências, decorrente de medidas excessivamente altas para rupturas gagas para um dos indivíduos do Grupo 1, favorecendo a diferença com significância estatística.

\section{DISCUSSÃO}

O presente estudo propôs a investigação de aspectos lingüísticos e paralingüísticos, verbais e não-verbais com o propósito de estabelecer o perfil comunicativo de indivíduos com a SWB sob uma perspectiva pragmática, abrangendo a categoria de habilidades conversacionais.

Esta perspectiva avaliativa da linguagem justifica-se uma vez que se mostra sensível à detecção de dificuldades pragmáticas, por visualizar a funcionalidade da comunicação pela sua totalidade e não apenas pelos componentes lingüísticos isolados ${ }^{(6,19,21-22)}$.

A hipótese levantada para este estudo pôde ser comprovada uma vez que os achados apontaram para a existência de diferenças importantes no desempenho comunicativo dos indivíduos com a SWB quando comparados a outros indivíduos de mesma idade mental com ausência de dificuldades de linguagem/aprendizagem.

A comparação de desempenho entre os dois grupos que diferiram quanto à presença ou não da síndrome genética, mas com idade mental semelhante, é um recurso metodológico freqüentemente empregado em estudos internacionais ${ }^{(4-5,7,11-13)}$, na tentativa de entender melhor o desempenho comunicativo de indivíduos com esta condição genética e, também, com o propósito de excluir a interferência dos mecanismos cognitivos nas possíveis dificuldades comunicativas encontradas no Grupo 1.

A literatura especializada nesta área, freqüentemente discorre sobre a suposta dissociação de aspectos cognitivos e lingüísticos nesta síndrome ${ }^{(4-5)}$. A este respeito, os achados deste estudo, ainda que tímidos para uma análise mais aprofundada, foram condizentes com estudos anteriores que ressaltaram a interferência no desempenho lingüístico dos indivíduos com a SWB, dependendo da complexidade de tarefas lingǘsticas utilizadas no processo avaliativo ${ }^{(5,17,19)}$. Conforme o estudo mostrou, a partir da estratégia de conversação adotada, foram identificados prejuízos lingüísticos tão graves quanto às limitações cognitivas apresentadas pelo indivíduo com a SWB ${ }^{(4,13,19)}$.

Um aspecto importante a ser destacado é a característica sociável, freqüentemente presente nos indivíduos com a SWB. Esta característica sugere ser reflexo da intencionalidade comunicativa, que parece fazer parte da natureza desses indivíduos, de forma que se envolvem facilmente em uma situação de comunicação. Porém, este comportamento sociável pode ser considerado ambíguo uma vez que favorece também a transgressão de regras sócio-comunicativas ${ }^{(14)}$, que abrangem a organização formal da conversação e a capacidade de manutenção e desenvolvimento temático.

Dentre os prejuízos lingüísticos graves do ponto de vista sócio-comunicativo, foram identificados no Grupo 1, com a SWB, comprometimentos quanto à organização formal das conversações com significativas restrições quanto ao cum- primento de regras para manter e adotar turnos. Essa dificuldade sugeriu estar relacionada com o comportamento impulsivo - que freqüentemente ocasiona a interrupção do turno do interlocutor - que resulta na dificuldade para aguardar sua vez e no uso freqüente de respostas precipitadas. No que diz respeito à persistência temática durante o intercâmbio conversacional, esta foi uma característica recorrente entre os indivíduos com a SWB. Tal característica sinalizou a presença de limitações lingüísticas decorrentes de restrições lexicais, uma vez que apresentaram dificuldades para introduzirem novos temas, como também no uso de estratégias lingüísticas que sinalizam a retomada ao tema com a finalidade de manter a coerência e o intercâmbio conversacional ${ }^{(14)}$, o que resultou no alto índice de iniciativas e respostas inadequadas em relação ao Grupo 2.

Em alguns casos com a SWB, na tentativa de prosseguir com a interação comunicativa, foram observados comportamentos verbais do tipo ecolalia e perseveração $o^{(6)}$, que embora não tenham sido características recorrentes aos indivíduos com a síndrome, também conferiram preenchimento do espaço comunicativo. Esses comportamentos representaram um impacto negativo do ponto de vista funcional da linguagem, uma vez que resultaram na impossibilidade do interlocutor compartilhar da informação a fim de continuar com o processo conversacional.

A dificuldade para atingir os objetivos comunicativos durante a conversação, sugeriu ser o motivo pelo qual a freqüência de turnos comunicativos foi superior para o Grupo 1 em comparação ao Grupo 2. Notou-se uma relação direta entre a freqüência aumentada para a troca de turno e a EME inferior, para o grupo de indivíduos com a SWB. Este achado corrobora estudo anterior ${ }^{(14)}$ que atribuiu a mudança rápida de turno às limitações que os indivíduos com a SWB apresentam para produzir respostas contingentes e colaborativas às informações do interlocutor, requerendo a mudança de turno por parte do interlocutor, na tentativa de prosseguir com a interação. Sendo assim, se por um lado há restrições no que diz respeito à quantidade e qualidade de informação, por outro, é possível entender que os enunciados produzidos pelos indivíduos com a SWB também são limitados no que diz respeito ao número de palavras utilizadas para compor o enunciado, como mostrou a medida referente à EME.

Nossos achados também mostraram que a característica de fala precisa e inteligível pode ser variável nesta síndrome, discordando dos resultados apresentados por alguns pesquisadores que pontuaram a integridade do sistema fonológico para esses indivíduos ${ }^{(6-8)}$. Este estudo mostrou que a ininteligibilidade de fala foi um dos itens com diferença estatística significante entre os grupos, junto com a persistência temática, que prejudicou tanto a ocorrência de iniciativas quanto a de respostas durante a conversação, corroborando a presença de prejuízos tanto no processamento semântico quanto fonológico para os indivíduos com a SWB ${ }^{(9-}$ 10). Destaca-se aqui a ocorrência de alterações fonológicas para os casos com a SWB com rebaixamento intelectual significativo, segundo desempenho nos testes psicométricos utilizados para este estudo.

Esses achados constituem evidências de problemas no 
processamento lingüístico da informação e não unicamente cognitivo. Assim, é possível dizer, que em parte, os achados deste estudo corroboram a presença de prejuízos lingüísticos típicos dos distúrbios semântico-pragmáticos da linguagem nos indivíduos com a SWB ${ }^{(14)}$, destacando-se os casos avaliados que apresentaram desempenho cognitivo limítrofe ou dentro da média inferior de normalidade, mas que ainda assim, apresentaram alterações significativas nos aspectos léxico-semânticos, com integridade do sistema fonológico e relativa superioridade no domínio sintático.

Embora a sintaxe tenha sido uma habilidade mais favorável se comparada ao domínio de habilidades léxico-semânticas - para esses casos com a SWB em que o desempenho cognitivo esteve próximo aos padrões de normalidade - esta habilidade está distante de ser considerada íntegra quando comparada aos indivíduos com mesma idade mental avaliados neste estudo. Esses achados estão próximos aos que foram encontrados em estudos que notaram a presença de dificuldades para domínios sintáticos mais complexos ${ }^{(5,10-11)}$, destacando um estudo brasileiro nesta área ${ }^{(12)}$. Foi fato notório para os indivíduos com a SWB com grave comprometimento cognitivo a presença de prejuízo sintático mais grave, assim como para os demais domínios lingüísticos que abrangem a semântica, a fonologia e a pragmática.

A evidência de um desempenho sintático menos favorável para os indivíduos com a SWB em nosso estudo - contrapondo aos achados da literatura, que descreveram a integridade para domínios sintáticos da linguagem nesta síndrome ${ }^{(4)}$ - pode estar relacionada com o nível organizacional dos sistemas de línguas, dentre as quais a língua portuguesa pode ser considerada uma língua complexa. Tal argumentação também foi sugerida em outro estudo ${ }^{(13)}$ no qual os autores pontuaram prejuízos sintáticos em sujeitos com a SWB, falantes do Italiano, língua essa considerada complexa do ponto de vista normativo.

Desta forma, a presença de prejuízos comunicativos apresentados por esses indivíduos, envolvendo tanto domínios lingüísticos estruturais quanto funcionais pode ser decorrente de dificuldades específicas de linguagem que podem vir associadas a prejuízos de funções cognitivas. Esta afirmação pode ser sustentada pelos achados deste estudo, uma vez que foi notória a presença de prejuízos comunicativos que foram recorrentes, porém, variáveis quanto ao grau de comprometimento para os indivíduos com a síndrome, que não apresentaram déficit cognitivo se comparados àqueles com deficiência intelectual grave.

Assim como outros estudos já haviam apontado para o uso da prosódia, como um recurso comunicativo que favorece a funcionalidade da linguagem dos indivíduos com a $\mathrm{SWB}^{(15-17)}$, este estudo também mostrou que freqüientemente esses indivíduos utilizaram estratégias comunicativas - que não se restringiram ao domínio paralingüístico, mas também lingüístico - na tentativa de suprimir suas limitações cognitivas e de linguagem. Este fato pôde ser constatado pelo uso de clichês, recursos de efeito sonoro, recursos entonacionais e a persistência temática, observados durante a conversação ${ }^{(15-16)}$. Tais características comunicativas reforçaram a idéia de que esses indivíduos apresentaram um esti- lo de comunicação próprio com forte impacto sócio-comunicativo $^{(15)}$, o que sugeriu ser uma forte justificativa para serem conhecidos como Cocktail Party Speech (CPS).

$\mathrm{O}$ uso dessas estratégias comunicativas refletiu de forma positiva sobre a funcionalidade da linguagem dos indivíduos com a SWB, uma vez que lhes conferiu uma "aparente" competência para se comunicar ${ }^{(15-17)}$. Tal competência mostrou estar relacionada ao uso de informações que preenchem o espaço comunicativo, como as expressões idiomáticas, os trocadilhos e jogos verbais, principalmente quando associada à integridade do sistema fonológico. Esse conjunto de habilidades lingüísticas parece justificar o reconhecimento desses indivíduos como "fluentes"(6-8).

Os achados mostraram também que o uso de um instrumento objetivo para a avaliação da fluência da fala ${ }^{(28)}$, adotado por esta pesquisa, a partir do levantamento quanto à freqüência e classificação da tipologia de disfluências da fala, foi um recurso metodológico interessante que permitiu obter parâmetros sobre a efetividade da linguagem dos indivíduos com a SWB, recurso este que pôde ser reforçado pela análise estatística comparativa entre os indivíduos com e sem a síndrome.

A este respeito, foi possível notar, que as rupturas da fala identificadas nos indivíduos com a SWB não tendem a ser sugestivas de gagueira, como também foi apresentado pelo grupo sem a síndrome. A diferença estatística encontrada entre a comparação dos grupos esteve relacionada a uma assimetria na distribuição de freqüências, decorrente de medidas excessivamente altas para rupturas gagas para um dos indivíduos com a SWB. Em relação à análise estatística comparativa entre os grupos, notou-se uma porcentagem de rupturas comuns da fala mais elevada para o Grupo 1, o que pode ser reflexo de prejuízos linguíísticos dos indivíduos com a SWB.

A classificação das pausas observadas a partir da investigação da fluência da fala mostrou que os indivíduos com a SWB quando comparados aos indivíduos sem a síndrome, apresentou tendência ao aumento da freqüência de pausas plenas no discurso, do tipo partículas de codificação e de repetições de palavras. As pausas plenas proporcionaram a esses indivíduos com a SWB o preenchimento verbal durante a conversação, conferindo falsa idéia de continuidade do discurso. A inserção desses segmentos na fala ocasionou, na verdade, a interrupção na linearidade semântica, o que sugeriu neste estudo a presença de prejuízos na fluência verbal decorrente de comprometimentos léxico-semântico ${ }^{(4,7-8)}$.

Desta forma, o uso dos componentes paralingüísticos como os trocadilhos, os jogos verbais, a prosódia ${ }^{(15-16)}$ e as pausas plenas, também apontadas por este estudo, podem estar relacionados não somente ao grau de envolvimento na situação comunicativa dos indivíduos com a SWB, como também com a personalidade sociável e "falante" que nem sempre condiz com sua competência lingüística, conforme mostramos.

De um modo geral, nossos achados mostraram que o perfil comunicativo dos indivíduos com a SWB avaliados por este estudo, corroborou a idéia da existência de um quadro cognitivo e lingüístico heterogêneo ${ }^{(15,18-20)}$. Ficou claro que esses indivíduos apresentaram características comunicativas 
recorrentes entre si, que se diferenciam apenas quanto ao grau de comprometimento, sugerindo estar relacionada ao prejuízo cognitivo apresentado pelo indivíduo.

\section{CONCLUSÃO}

Este estudo mostrou que o perfil comunicativo dos indivíduos com a SWB frequientemente é caracterizado pela facilidade que apresentam para interagirem em uma situação de comunicação e que, com isso, buscam estratégias comunicativas como o uso de clichês, efeitos sonoros, recursos entonacionais e as pausas plenas na tentativa de preencherem o espaço comunicativo estabelecido com um interlocutor. Esses "artefatos comunicativos", nem sempre se mostraram favoráveis do ponto de vista sócio-comunicativo, como a presença de comportamentos verbais ecolálicos e perseverativos. Embora esses recursos representem impactos sócio-comunicativos distintos, ambos os casos, ou seja, tanto os indivíduos que utilizaram freqüentemente os clichês, recursos sonoros e prosódicos como também aqueles que apresentaram comportamentos verbais ecolálicos e perseverativos, são resultados das limitações lingüísticas, sejam elas no que dizem respeito aos aspectos estruturais ou funcionais da linguagem.
Embora a proposta deste estudo tenha sido a de traçar um perfil comunicativo para a população com a SWB investigada, não se pode negar que os achados desta pesquisa revelaram a existência de diferenças comunicativas interessantes quando o desempenho do Grupo 1, com a SWB, foi comparado ao Grupo 2, sem a síndrome e com idade mental semelhante à do Grupo 1. O desempenho comunicativo mais prejudicado do grupo com a SWB permitiu especular que comprometimentos lingüísticos nesta condição podem estar presentes, independente de diferença entre idade cronológica e mental. Estudos mais específicos, aumentando a casuística e reduzindo as diferenças de idade cronológica, para melhor comparar o desempenho comunicativo, são necessários para aprofundar o tema e subsidiar propostas avaliativas e de intervenção fonoaudiológica mais adequadas para esses indivíduos com a SWB.

Estudos mais abrangentes com esta população - comparada também com outras que apresentem alterações cognitivas como parte do fenótipo - podem não somente responder ao questionamento em torno da dissociação de habilidades cognitivas e lingüísticas nesta síndrome, como também favorecer os estudos em geral que abordam esta complexa correlação, em meio aos distúrbios da comunicação humana.

\begin{abstract}
Purpose: To describe the communicative profile of individuals with Williams-Beuren syndrome. Methods: The sample comprised 12 subjects with the syndrome, with chronological ages between 6;6 and 23;6 (Group 1), that were compared to other 12 subjects without the syndrome matched by mental age who had no language/learning difficulties (Group 2). The subjects were evaluated during conversational situation in order to classify verbal and non-verbal behaviors according to pragmatic criteria, number of turns per minute, sentences per turn, Mean Length Utterances, frequency and type of speech disfluencies, and classification regarding the type of full pauses of the speech. Results: The communicative profile of Group 1 showed easiness to interact during communication situations that involved the presence of variable structural and functional linguistic limitations, when compared to the subjects of Group 2. The subjects of Group 1 frequently used communicative strategies in the attempt to fill in the communication space, making use of clichés, sound effects, intonation resources and the full pauses, which proved to be favorable from the sociocommunicative's point of view, whereas echolalic and perseverative verbal behaviors can harm the communicative performance of these individuals. Conclusion: Group 1's worse communicative performance suggests that linguistic disabilities can be found in this condition, independently from the difference between chronological and mental age. Wider studies could allow an answer to the question of whether or not cognitive and linguistic abilities are dissociated in this syndrome, as well as to how complex is such correlation among human communication impairments.
\end{abstract}

KEYWORDS: Cognition; Communication; Language; Williams syndrome

\title{
REFERÊNCIAS
}

1. Meyer-Lindenberg A, Mervis CB, Berman KF. Neural mechanisms in Williams syndrome: a unique window to genetic influences on cognition and behaviour. Nat Rev Neurosci. 2006;7(5):380-93.

2. Stromme P, Bjornstad PG, Ramstad K. Prevalence estimation of Williams syndrome. J Child Neurol. 2002;17(4):269-71.

3. Morris CA, Mervis CB. Williams syndrome and related disorders. Annu Rev Genomics Hum Genet. 2000;1:461-84.

4. Bellugi U, Lichtenberger L, Jones W, Lai Z, St George M. I. The neurocognitive profile of Williams syndrome: a complex pattern of strengths and weaknesses. J Cogn Neurosci. 2000;12(Suppl 1):7-29.
5. Mervis CB, Robinson BF, Bertrand J, Morris CA, Klein-Tasman BP, Armstrong SC. The Williams syndrome cognitive profile. Brain Cogn. 2000;44(3):604-28.

6. Udwin O, Yule W. Expressive language of children with Williams syndrome. Am J Med Genet Suppl. 1990;6:108-14.

7. Vicari S, Bellucci S, Carlesimo GA. Procedural learning deficit in children with Williams syndrome. Neuropsychologia. 2001;39(7):665-77.

8. Somerville MJ, Mervis CB, Young EJ, Seo EJ, del Campo M, Bamforth $\mathrm{S}$, et al. Severe expressive-language delay related to duplication of the Williams-Beuren locus. N Engl J Med. 2005;353(16):1694-701. 
9. Laing E, Grant J, Thomas M, Parmigiani C, Ewing S, Karmiloff-Smith A. Love is... an abstract word: the influence of lexical semantics on verbal short-term memory in Williams syndrome. Cortex. 2005;41(2):169-79.

10. Brock J, McCormack T, Boucher J. Probed serial recall in Williams syndrome: lexical influences on phonological short-term memory. J Speech Lang Hear Res. 2005;48(2):360-71.

11. Clahsen H, Almazan M. Syntax and morphology in Williams syndrome. Cognition. 1998;68(3):167-98.

12. Freitas MC, Silveira MS, Corrêa LMS. Seletividade no desempenho lingüístico de portadores da síndrome de Williams e do déficit especificamente lingüístico (DEL). In: $3^{\circ}$ Congresso Internacional da Associação Brasileira de Lingüística; 2003, Rio de Janeiro. Anais. Rio de Janeiro: Pontifícia Universidade Católica do Rio de Janeiro; 2003. p.10-9.

13. Karmiloff-Smith A, Tyler LK, Voice K, Sims K, Udwin O, Howlin P, Davies M. Linguistic dissociations in Williams syndrome: evaluating receptive syntax in on-line and off-line tasks. Neuropsychologia. 1998;36(4):343-51.

14. Laws G, Bishop D. Pragmatic language impairment and social deficits in Williams syndrome: a comparison with Down's syndrome and specific language impairment. Int $\mathrm{J}$ Lang Commun Disord. 2004;39(1):45-64

15. Gonçalvez OF, Pérez A, Henriques M, Prieto M, Lima MR, Siebert MF, Sousa N. Funcionamento cognitivo e produção narrativa na síndrome de Williams: congruência ou dissociação neurocognitiva? Int J Clin Health Psychol. 2004;4(3):623-38.

16. Reilly J, Losh M, Bellugi U, Wulfeck B. "Frog, where are you?" Narratives in children with specific language impairment, early focal brain injury, and Williams syndrome. Brain Lang. 2004;88(2):229-47.

17. Tarling K, Perkins MR, Stojanovik V. Conversational success in Williams syndrome: communication in the face of cognitive and linguistic limitations. Clin Linguist Phon. 2006;20(7-8):583-90.
18. Catterall C, Howard S, Stojanovik V, Szczerbinski M, Wells B. Investigating prosodic ability in Williams syndrome. Clin Linguist Phon. 2006;20(7-8):531-8.

19. Stojanovick V, Perkins M, Howard S. Linguistic heterogeneity in Williams syndrome. Clin Liguist Phon. 2006; 20(7-8):547-52.

20. Vicari S, Carlesimo GA. Short-term memory deficits are not uniform in Down and Williams syndromes. Neuropsychol Rev. 2006;16(2):8794.

21. Cascella PW. Standardised speech-language tests and students with intellectual disability: a review of normative data. J Intellect Dev Disabil. 2006;31(2):120-4

22. Adams C, Lloyd J. Elicited and spontaneous communicative functions and stability of conversational measures with children who have pragmatic language impairments. Int J Lang Commun Disord. 2005;40(3):333-47.

23. Brasil. Ministério da Saúde. Conselho Nacional de Saúde. Diretrizes e normas regulamentadoras sobre pesquisas envolvendo seres humanos: resolução 196/96. Brasília: Conselho Nacional de Saúde; 1996.

24. Wechsler D. Wechsler Intelligence Scale for Children (WISC). 3rd ed. San Antonio: Psychological Corporation; 1991.

25. Wechsler D. WPPSI-R: Wechsler Preschool and Primary Scale of Intelligence - Revised. San Antonio: Psychological Corporation; 1989.

26. Wechsler D. WAIS-III: administration and scoring manual. San Antonio: Psychological Corporation; 1997.

27. Prutting CA, Kirchner DM. A clinical appraisal of the pragmatic aspects of language. J Speech Hear Disord. 1987;52(2):105-19.

28. Andrade CRF. Fluência. In: Andrade CRF, Befi-Lopes DM, Fernandes FDM, Wertzner HF. ABFW: teste de linguagem infantil nas áreas de fonologia, vocabulário, fluência e pragmática. Carapicuíba: Pró-Fono; 2000. p. 61-76. 